\title{
El Síndrome de Fatiga Crónica: del modelo de susceptibilidad a la hipótesis de la cadena de estrés en el área laboral
}

\section{RESUMEN}

El modelo de susceptibilidad tiene en cuenta la combinación de algunos elementos: la susceptibilidad biológica, la susceptibilidad al mobbing, la susceptibilidad al burn-out, la susceptibilidad al estrés laboral, y los factores de protección como: apoyo social, autoconcepto positivo, locus de control interno, alta autoestima y sentimiento de autoeficacia.

La hipótesis de la cadena de estrés en el área laboral considera que la persistencia del estrés laboral es acumulativa. El mobbing, el síndrome de burn-out y el estrés laboral provocan distintos tipos de fatiga. Si los factores estresantes que generan estos problemas perseveran durante tiempo, la fatiga puede devenir en lo que se ha dado en llamar síndrome de fatiga crónica.

Palabras Clave: Síndrome de Fatiga Crónica, Mobbing, Burn-out, Estrés Laboral, Autoconcepto, Autoestima, Locus de Control, Autoeficacia, Apoyo Social.

\section{SUMMARY}

The susceptibility model has to be with the mixture of different elements: the biological susceptibility, the susceptibility to mobbing, the susceptibility to burn-out, the susceptibility to job stress and the protection factors such as: the social support, the positive self-concept, the internal locus of control, the high level of self-esteem and the self-efficacy feeling.

The hypothesis of stress chain in the job area afirms that the persistance of job stress is acumulative. The mobbing, the burn-out syndrome, the job stress provoke different kinds of fatigue. If the stress factors, which lead to these problems, waist too much time, the fatigue may come to be what is called Chronic Fatigue Syndrome.

Key Words: Chronic Fatigue Syndrome, Mobbing, Burn-out, Job Stress, Self-concept, Self-esteem, Locus of Control, Self-efficacy, Social Support. 


\section{Introducción}

Aunque no se conoce la etiología del Síndrome de Fatiga Crónica (SFC) se han sugerido diversas hipótesis etiopatogénicas, quizá porque este síndrome sea de origen heterogéneo.

Así, se han contemplado los factores virológicos, entre los cuales, el que ha ocupado el primer lugar ha sido el virus Epstein Barr, responsable de la mononucleosis infecciosa. Actualmente se estudian otros virus, como el citomegalovirus, los enterovirus y los retrovirus.

Bajo otro prisma, se ha señalado que el SFC sería una variante atípica de un trastorno psiquiátrico, debido a su frecuente asociación con la depresión mayor y con trastornos de ansiedad.

Desde otra óptica, se ha observado que en el SFC existen indicios que denotan un compromiso del sistema inmune (manifestaciones alérgicas como laringitis y bronquitis).

Por otro lado, algunas investigaciones sostienen que podría existir una disfunción hipotalámica con una disminución de la actividad de la corticotrofina y del cortisol basal. También se ha sugerido que habría un aumento de la sensibilidad de los receptores serotoninérgicos hipotalámicos. Incluso se ha postulado como causa una alteración primaria del sueño (1).

De cualquier modo, el diagnóstico es difícil porque no se dispone de pruebas de laboratorio y, por tanto, se establece fundamentalmente a través de los síntomas descritos por los pacientes (2).

\section{Modelo de susceptibilidad}

El modelo de susceptibilidad (Figura 1) comparte la característica de ser multifactorial, pues considera aspectos biológicos, psicológicos y laborales. Postula que cada individuo tiene un distinto nivel de vulnerabilidad para llegar al SFC.

Este modelo establece la combinación de algunos elementos: susceptibilidad biológica, susceptibilidad al mobbing, susceptibilidad al burn-out, susceptibilidad al estrés laboral, y los factores de protección como: autoconcepto positivo, alta autoestima, locus de control interno, sentimiento de autoeficacia y apoyo social.

Figura 1. MODELO DE SUSCEPTIBILIDAD

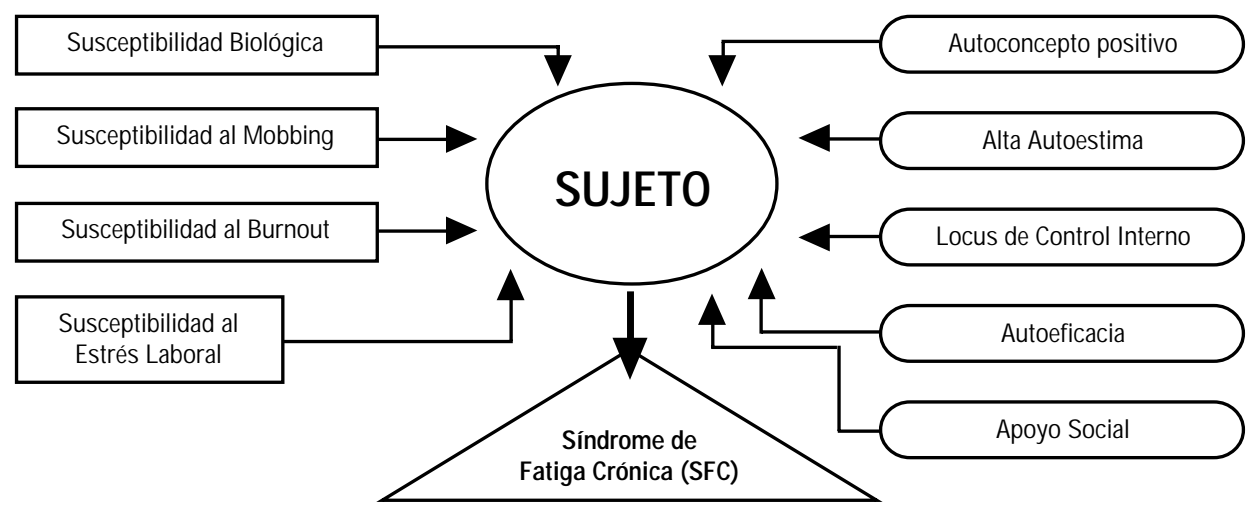


El mobbing podría considerarse como una forma de estrés laboral que presenta la particularidad de que no ocurre por causas directamente relacionadas con el desempeño del trabajo o con su organización sino que tiene su origen en las relaciones interpersonales que se establecen en cualquier empresa entre los distintos individuos.

Se ha afirmado que el mobbing es el encadenamiento a lo largo de un período de tiempo bastante corto de intentos o acciones hostiles consumadas, expresadas o manifestadas por una o varias personas hacia una tercera (3).

Otros han manifestado que el mobbing es el continuo y deliberado maltrato verbal y moral que recibe un trabajador por parte de otro u otros, que se comportan con él cruelmente con vistas a lograr su destrucción psicológica y a obtener su salida de la organización a través de diferentes procedimientos (4).

Unos identifican en las consecuencias del mobbing una sintomatología que incluye cansancio, nerviosismo, problemas de sueño, migraña, trastornos digestivos, lumbalgias y estado depresivo (5). Otros señalan ansiedad, baja autoestima, apatía, sentimientos de fracaso, distorsiones cognitivas, adicción, conductas de aislamiento, patología psicosomática (dolores, trastornos funcionales y orgánicos), inadaptación social, hipersensibilidad a la crítica, cuadros depresivos, paranoia y autolisis (6).

Se ha señalado que los individuos de riesgo para sufrir el mobbing son aquellos sujetos con ciertos rasgos depresivos, con baja autoestima, con tendencia a autoculpabilizarse y están necesitados de afecto y aprobación constante, en definitiva dan la impresión de ser inofensivos y de encontrarse indefensos (7).

Por otro lado, se ha considerado que el Síndrome de Burn-out hace referencia a un síndrome de cansancio o agotamiento emocional, despersonalización o deshumanización y falta o disminución de realización personal que puede ocurrir entre individuos que trabajan con personas. Es decir, el Síndrome de Burn-out aparece en el individuo como una consecuencia de trabajar en condiciones difíciles en contacto directo con usuarios, pacientes o clientes y que tiene resultados negativos para la persona y para la Institución (8).

El cansancio emocional se refiere a la disminución y pérdida de recursos emocionales, junto con la sensación de que uno ya no tiene nada que ofrecer psicológicamente a los demás. Así como sentimientos de estar emocionalmente agotado y exhausto debido al trabajo que el sujeto realiza. La despersonalización consiste en el desarrollo de una actitud insensible hacia las personas con quienes se trabaja. $\mathrm{O}$ sea, una actitud negativa en las respuestas hacia los beneficiarios del propio trabajo. Y la falta de realización personal es la tendencia a devaluar el propio trabajo, reprochándose no haber alcanzado los objetivos propuestos, con vivencias de insuficiencia personal y baja autoestima. Es decir, sentimientos negativos sobre la realización personal, que supone infravaloración hacia uno mismo y hacia el trabajo, evitación de las relaciones interpersonales y profesionales, baja productividad e incapacidad para soportar la presión (9). 
Se ha observado que son susceptibles de burnout las personalidades emotivas, es decir, aquellos que tienen más desarrollada la sensibilidad para temas relacionados con el trato humano (10), los llamados «activistas sociales» que ofrecen ayuda a los demás y los calificados de «visionarios» porque toman su labor más como una cruzada que como una tarea para ganarse la vida, además suelen ser personas autoexigentes, con baja tolerancia al fracaso y perfecionistas (11).

De otra parte, se ha definido el estrés laboral como una inadaptación al trabajo, como la interacción desfavorable entre los atributos del trabajador y las condiciones de trabajo que conducen a trastornos psicológicos y a conductas insanas y finalmente a la enfermedad (12).

Así, se ha establecido que las experiencias de estrés laboral surgen cuando las demandas del trabajo son altas, y al mismo tiempo, la capacidad de control de la misma es baja (13).

Otros han señalado que el desencadenante de estrés es el desajuste entre las necesidades, deseos o expectativas y la situación real (14).

En todo caso, el estrés laboral es un conjunto de reacciones emocionales, cognitivas, fisiológicas y del comportamiento a ciertos aspectos adversos o nocivos del contenido, la organización o el entorno del trabajo.

Se han investigado distintos estresores en el trabajo, y estos van desde el ambiente físico (ruido, contaminación, iluminación, temperatura...) a la estructura y política organizacional (ambigüedad de rol, conflicto de rol, promoción y desarrollo profesional) pasando por el contenido de la tarea (sobrecarga laboral, baja participación en las decisiones, presión horaria, sobreutilización de las capacidades...).

Las consecuencias negativas del estrés laboral pueden reflejarse en problemas cardiovasculares, en patología gastrointestinal, crisis de nervios (15), trastornos musculares, depresión, cáncer, úlceras, drogodependencia, suicidio (16) y patología dermatológica.

Son más susceptibles al estrés laboral los sujetos dependientes, aquellos que tienen un patrón de conducta tipo A (activos, enérgico, competitivos, ambiciosos, agresivos, impacientes y diligentes), los de patrón de conducta tipo $\mathrm{C}$ (es decir, aquellos que no exteriorizan sus emociones, que procuran complacer a los demás, a pesar de sacrificar sus propios objetivos y que evitan la confrontación) (17).

Entre los factores de protección hay que señalar que aunque en la delimitación del autoconcepto y la autoestima hay cierta controversia, porque ambos términos se suelen utilizar indistintamente para referirse al conocimiento que la persona tiene de sí misma (18). Es conveniente diferenciarlos, porque el autoconcepto corresponde a cómo se percibe uno a sí mismo y la autoestima a cómo se siente uno consigo mismo. Así, en el autoconcepto prima la dimensión cognitiva, mientras que en la autoestima prevalece la valorativa (19).

$\mathrm{El}$ autoconcepto es la percepción o imagen de uno mismo. Es decir, el conjunto de pensamientos con los que nos describimos a nosotros mismos (19). No es algo innato sino que se forma a partir de los sentimientos y experiencias, y sufre 
cambios favorecidos por las diferentes variables ambientales y personales, las cuales determinan que el individuo tenga un mejor o peor ajuste a periodos concretos del ciclo vital (20).

El autoconcepto es multidimensional (interacción familiar, interacción con iguales, labilidad emocional, self social, integración escolar, logro académico, self físico, deporte) y multifacético de estructuras que desempeñan un papel primordial en la organización de la conducta (21). En todo caso el autoconcepto es la visión que uno tiene de sí mismo en un tiempo particular (22).

Se ha relacionado el autoconcepto positivo con un estilo atributivo interno (23), con una mayor participación deportiva (24) y como elemento activo y dinámico en aspectos conductuales y afectivos (25).

El autoconcepto es uno de los principales determinantes de la conducta y un factor que garantiza la salud mental de la persona (26). Ha sido implicado en la motivación, en la regulación de la conducta (27), en los procesos de cambio y en las relaciones interpersonales (28).

Se ha intentado definir la autoestima desde distintos puntos de vista. Por un lado, se ha basado en la idea de que el self puede ser tratado como un objeto de atención, como cualquier cosa o posibilidad. Por otro lado, se ha definido como la respuesta psicológica que la persona tiene de su self de naturaleza afectiva o en el sentimiento negativo o positivo; o de aceptación o rechazo (29).

De cualquier modo, la autoestima es la evaluación que efectúa y mantiene el individuo en referencia a sí mismo, y expresa una actitud de aprobación o de desaprobación (30). Además el grado de autoestima viene determinado por la relación entre la imagen real que cada uno tiene de sí mismo, es decir, cómo es, y la imagen ideal, cómo le gustaría ser (31).

Por tanto, el autoconcepto sería la representación mental que el sujeto hace de sí mismo y la autoestima vendría a ser la dimensión evaluativa de esa representación (32).

El locus de control (33) es un constructo relacionado con la capacidad que tiene un sujeto para controlar el medio que le rodea. Y hace referencia a las creencias que tiene un sujeto sobre si es su conducta o un factor fuera de control, lo que determina aquello que le ocurre a la persona (34).

De manera que cuando el individuo cree que es capaz de llevar a cabo una conducta que le conducirá a su objetivo decimos que su locus de control es interno. Éste se ha relacionado con un buen estado psicoafectivo (35). Por el contrario, cuando la causa de su conducta la sitúa en factores del exterior como el azar, la suerte o el destino, su locus de control tiende a ser externo (25).

El locus de control es un concepto estrechamente vinculado con la autoeficacia, lo que sugiere que las medidas futuras de control interno y externo deben corresponderse con la medida presente de autoeficacia en relación con una conducta específica (36).

La autoeficacia es una variable mediadora que condiciona la conducta, el esfuerzo y el resultado, independientemente 
de la capacidad del sujeto. Y se entiende como la creencia relacionada con la confianza que tiene un sujeto para realizar ciertas conductas (37).

El concepto de apoyo social ha sido utilizado en muy diversos sentidos, como red social, como contactos sociales significativos y como posibilidad de tener personas confidentes a las que se puede expresar sentimientos íntimos.

También se han definido los sistemas de apoyo social como los vínculos entre individuos y grupos que sirven para improvisar competencias adaptativas en el manejo de los desafíos y problemas inmediatos, en crisis y en privaciones (38).

Se han distinguido cuatro tipos de apoyos: emocionales, instrumentales, de información y de valoración (39). Aunque para otros autores, el apoyo social está integrado por empatía, estímulo, información, ayuda material y expresiones de participación (40).

Es necesario destacar la importancia de la percepción del apoyo social, porque la realidad para el individuo es fundamentalmente la realidad percibida.

El apoyo percibido se puede identificar como la información que llega a tener una persona de una o más de las tres siguientes clases: información de que el sujeto es querido, información de que el sujeto es estimado y valorado e información de que el sujeto posee capacidad para establecer redes de comunicación y de relación (41)

El concepto de apoyo social, aunque paralelo al de red social no debe considerarse sinónimo de éste.
La estructura de la red social hace referencia al modelo de relaciones que constituyen el entramado social de la persona y los miembros de su red. Se han distinguido varios aspectos de la estructura: tamaño, densidad, proximidad, tipo e intensidad de las relaciones (42).

La red social típica de un individuo consta de 6 a 10 personas a las que conoce íntimamente y que en su mayoría se conocen entre sí y aproximadamente otras 30 personas a las que ve con regularidad. La mayoría de los individuos se relacionan con cinco o seis grupos relativamente independientes formados por seis o siete personas con interacciones entre sí (43).

Otros estiman que una persona normal mantiene una relación consistente con veinticinco personas. Estas personas con las que uno se relaciona podrían estar situadas en cuatro subgrupos: familia, parientes, amigos y vecinos o compañeros de trabajo. Esta red social normal se caracteriza por una interacción frecuente, un afecto positivo y un componente instrumental de apoyo importante (44). Y ha prevalecido la idea de que las personas con muchas o muy estrechas relaciones, reciben más apoyo social que aquéllos que carecen de ellas.

\section{Hipótesis de la cadena de estrés laboral}

La hipótesis de la cadena de estrés en el área laboral considera que la persistencia del estrés laboral es acumulativa y puede atravesar tres estadios (Figura 2):

1. Mobbing o Síndrome de Burn-out o Estrés Laboral.

2. Fatiga Laboral: de la que puede recuperarse con descanso o cambio de trabajo. 


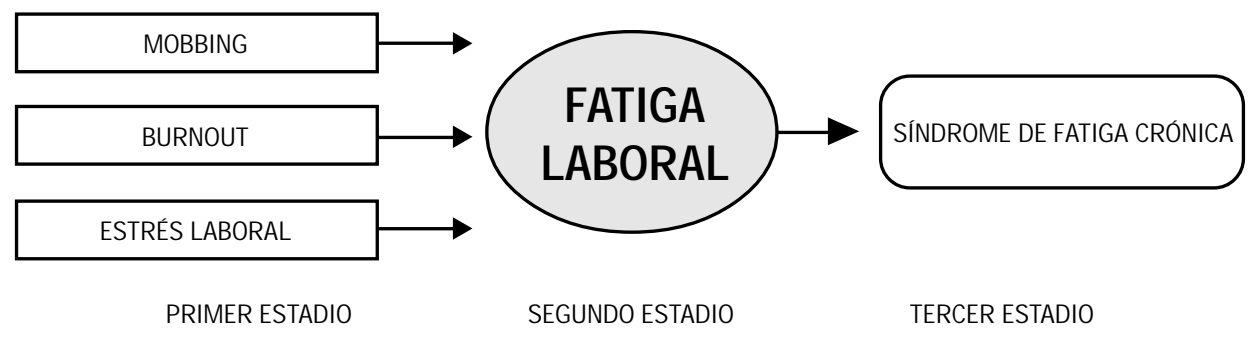

3. Síndrome de Fatiga Crónica (SFC) de la que no se recupera ni con tiempo de descanso ni con cambio de trabajo.

El acoso psicológico o mobbing, el síndrome de desgaste profesional o síndrome de burn-out y el estrés laboral provocan fatiga laboral. Si los estresores que generan estos problemas perseveran durante tiempo, la fatiga laboral podría devenir en el Síndrome de Fatiga Crónica (SFC).

Bajo el termino fatiga se han etiquetado estados de diferente intensidad y no es fácil dar con una definición única y aceptable para todos.

La persona que se somete a un esfuerzo intenso y prolongado sufre un progresivo debilitamiento de la capacidad de resistencia que le lleva a la fatiga (45). De manera que un prolongado esfuerzo físico o mental provoca un cambio en el organismo que tiene como resultado el declive en el rendimiento y el sentimiento de fatiga (46).

La fatiga es un mecanismo de seguridad fisiológico que indica al organismo la necesidad imperiosa de descanso y que surgirán cambios metabólicos que causa- rán daños irreversibles si se continua el esfuerzo. Las consecuencias pueden ser diversas: orgánicas, psicopatológicas, psicosomáticas, etc...

Se ha realizado una tipología de la fatiga laboral en función de la parte del organismo implicada: fatiga muscular, fatiga intelectual, fatiga nerviosa, fatiga psicológica y fatiga sensorial (47).

La fatiga muscular implica la imposibilidad de mantener un esfuerzo muscular. Este tipo de fatiga ha perdido protagonismo a favor del campo psíquico. Así la fatiga mental es más difícil de apreciar que la muscular y cuando aparece se evidencia por irritabilidad, ansiedad, cefalea, etc...

La fatiga mental ha sido descrita en la CIE-10 como la presencia desagradable de asociaciones o recuerdos que distraen al individuo, de dificultades para concentrarse y en general de falta de rendimiento del pensamiento.

El denominado SFC es una entidad clínica que se acuñó por un comité internacional de expertos en 1985 con la finalidad de delimitar y definir un conjunto de sintomatología diversa de curso crónico 
que giraba alrededor de una gran fatigabilidad al realizar mínimos esfuerzos (48).

Desde finales del siglo XIX se han venido describiendo síndromes caracterizados por una fatiga crónica debilitante, que han sido conocidos con distintos nombres en diferentes épocas, quizá por eso al SFC se la ha denominado «la enfermedad de los mil nombres» desde enfermos de sofá o de cama hasta el síndrome del ama de casa porque afectaba principalmente a mujeres de clase media, pasando por la enfermedad del yuppie ya que se la ha relacionado con personas sometidas a niveles altos de estrés o con una fuerte demanda laboral. Entre los factores predisponentes se han advertido el trabajo excesivo, el sedentarismo, los antecedentes de enfermedad vascular y la edad avanzada, aunque niños y adultos presentan síntomas similares (49).

Es conveniente ventilar si es una enfermedad emergente, a pesar de que este tipo de sintomatología o parecida ha existido en todo momento a lo largo de la historia de la humanidad. De hecho, se han descrito un número de enfermedades que tienen un espectro de síntomas similares a los del SFC, como el Síndrome Fibromiálgico, la Neurastenia, la Mononucleosis Crónica y el Síndrome de Fatiga Postviral, a las que la fatiga crónica está asociada. Se ha diferenciado de un trastorno similar denominado fibromialgia, que consiste en una profunda fatiga, mialgias con puntos dolorosos específicos, alteraciones del humor y sueño no reparador (1). En España, aunque no existen estadísticas fiables por la dificultad de realizar diag- nósticos la Asociación Catalana de afectados por el SFC estima que pueden padecer la enfermedad el 1 por 1000 de la población, lo que parece poco relevante si se compara con otros países occidentales que señalan que del 2 al $3 \%$ sufrirían el SFC (50).

Hasta el momento el SFC es un diagnóstico que se hace por exclusión (51) por la inespecificidad de los síntomas, porque la analítica generalmente es normal y las fluctuaciones inesperadas de los síntomas dificultan la labor para establecer un diagnóstico diferencial (52). Por eso, todavía hay cierto grado de controversia acerca del diagnóstico del SFC y se han realizado encuentros para ir aclarándolo. Así en 1990 en Gran Bretaña se definió el SFC como aquel en que la fatiga es el síntoma principal, pero debía de ser de comienzo definido y no de toda la vida. Esta fatiga tiene que ser severa e invalidante, durar más de 6 meses, afectar a las funciones físicas y mentales y cubrir más del $50 \%$ del tiempo. Además, la sintomatología consiste en mialgias, alteraciones del humor, del sueño y de la concentración y memoria.

Con la misma intención en 1994 un grupo internacional concluyó que el criterio de SFC se debía basar en la autoafirmación del paciente y en que la sintomatología estaría presente más de 6 meses. Se eliminó la exigencia de que causara una reducción de la actividad de más de un $50 \%$. También se eliminaron algunos criterios menores como la fiebre y se exigió la presencia de sólo 4 de los 8 criterios menores siguientes: Alteración de la memoria o concentración, odinofagia, ganglios linfáticos cervicales o axilares dolorosos, dolor muscular, poliartralgias, 
cefalea, alteraciones del sueño y malestar post-ejercicio (53).

Estos criterios permiten establecer si no un diagnóstico concreto sí un diagnóstico sindrómico.

La característica que define el SFC es la fatiga física y mental (54) que es descrita como una sensación de cansancio profundo y que incapacita para realizar las actividades diarias, generando alteraciones severas en la vida familiar, social y laboral. Esta sensación de fatiga se acompaña de malestar general que no mejora con el reposo.

Otro signo característico del SFC es que se presenta en forma de crisis con exacerbación de síntomas y con intensidad y duración variable, pero cada crisis suele ser diferente. $\mathrm{Y}$ en los periodos de intercrisis la sintomatología puede disminuir pero no desaparece (55).

En todo caso, el llamado SFC es una enfermedad crónica caracterizada por un estado general de cansancio tanto mental como físico y por presentar síntomas neurológicos (cefalea, pérdida de memoria e insomnio), psicopatológicos (ciclotimia, disminución de las funciones cognitivas y ataques de pánico), debilidad muscular, fatiga, sudoración nocturna, adenopatías, diarrea persistente, molestias faríngeas y dolores musculares o articulares (56).

\section{Conclusiones}

1. La terminología SFC puede ser blanda, por su falta de concreción, pero tiene el valor de intentar definir y asentar algunos criterios diagnósticos.
2. Sería conveniente que ante cada caso de SFC se valorara exhaustivamente el clima y relaciones laborales e incorporar el abordaje de este área en el tratamiento.

3. Aunque la etiopatogenia del SFC se desconoce se han propuesto diversas hipótesis entre las que están: factores víricos, trastorno psiquiátrico, disfunción hipotalámica y anomalía del sistema inmune.

4. El SFC consiste básicamente en una serie de síntomas que incluyen fatiga, cefalea, perturbaciones del sueño, dificultades de concentración y dolor muscular que tienen una evolución de más de 6 meses.

5. El SFC no es una enfermedad mortal pero cada día lleva a más personas a una situación que puede ir desde ligeras limitaciones hasta una invalidez funcional total.

6. Sería necesario ir perfilando ajustados criterios diagnósticos para el SFC que aún es un síndrome poco específico y que obliga al paciente a ir de médico en médico en busca de un diagnóstico, con objeto de que pudiera irse aceptando como enfermedad profesional. En España son frecuentes las dificultades para realizar el diagnóstico entre SFC y Fibromialgia. El diagnóstico diferencial podría estribar en que la fatiga sería profunda en el SFC y en la Fibromialgía resaltaría el dolor muscular, aunque no hay evidencia sobre el punto anterior las líneas de investigación en Fibromialgia se han dedicado a la patología muscular y en el SFC en la etiopatogénesis infecciosa y del sistema inmunológico. 


\section{BIBLIOGRAFÍA}

1. Natelson, B.H. Chronic Fatigue Syndrome. JAMA. 2001. 285: 2557-2559.

2. Whitting, P.; Bagnall, A.M.; Sowden, A.J.; Cornell, J.E.; Mulrow, C.D.; Ramirez, G. Intervenciones para el tratamiento y control del síndrome de fatiga crónica. JAMA 2002. 11, 3: 42-52.

3. Leymann, H. Mobbing. La persécution au travail. Du Seuil. París. 1996.

4. Piñuel y Zabala, I. Mobbing: Cómo sobrevivir al acoso psicológico en el trabajo. Sal Terrae. Santander. 2001.

5. Hirigoyen, M.F. El acoso moral en el trabajo. Paidós. Barcelona. 2001.

6. González de Rivera, J.L. El maltrato psicológico. Cómo defendersedel mobbing y otras formas de acoso. Espasa. Madrid. 2002.

7. Schuster, A. Mobbing, bullying and peer. American Psychological Association. www.apa.org/psa/julaug96/sb.html. 1-10.

8. Maslach, C.; Jackson, S.E. Maslach Burnout Inventory. Consulting Psychologist Press. Palo Alto. California. 1986.

9. Gil-Monte, P.; Peiró, J.M. Desgaste psíquico en el trabajo: El síndrome de quemarse. Síntesis. Madrid. 1997.

10. Garden, A.M. Burnout: The effect of psychological type on research finding. Journal Occupational Psychology.1989. 62: 223-234.

11. Chermis, C. Staff burnout: Job stress in the Human Service. Sage. Beverly Hills and London.1980.

12. Sauter, S.L; Murphy, L.R.; Hurrel, J.J. Prevention of workrelated psychological disoreder: A national strategy proposed by the National Institute for Occupational Safety and Health (NIOSH). American Psychologist. 1990. 45: 1146-1158.

13. Karasek, R.A. Job demands, job decision latitude, and mental strain. Implications for job redesign. Administrative Science Quarterly. 1997. 24: 285-308.

14. Edwards, J.R. The determinants and consequences of coping with stress. En L.C. Cooper y R. Payne (Eds.). Causes, coping and consequences of stress at work. John Wiley and Soons. New York. 1988.

15. Peiró, J.M. Desencadenantes del estrés laboral. Eudema. Madrid. 1992.

16. Fernández Montalvo, J.; Garrido, F. Psicopatología Laboral. Servicio de Publicaciones de la Universidad Pública de Navarra. Pamplona. 1999.

17. Fernández Abascal, E.; Jiménez, M.P. Control del Estrés. UNED Ediciones. 2002.

18. Watkins, D.; Dhawan, N. Do we need to distinguish the constructs of self-concept and self-esteem? Journal of Social Behavior and Personality. 1989. 4: 55-562.

19. Amador, J.A.; Forns, M. Autoestima. Cómo mejorar la propia imagen. Colimbo. Barcelona. 1995.

20. Torres Ortuño, A.I. Autoconcepto y calidad de vida en personas de edad: un estudio empírico. Anales de Psiquiatría. 1998. 14, (2): 56-85 y 14, (3): 116-132. 
21. Markus, H. Unresoved issues of self-representations. Cognitive Therapy and Research. 1990. 14, 2: 241-253.

22. Moore, B.; Fine, B. Psychoanalytic terms and concepts. Yale University Press. New Haven. 1990.

23. Marsh, H. Self-serving (¿bias?) in academic atributions: its relation to academic achievement and self-concept. Journal of Educational Psychology. 1986. 78: 190-200.

24. Schumaker, J.; small, L.; Wodd, J. Self-concept, academic achievement and athletic participation. Perceptual and Motor Skills. 1986. 62: 387-390.

25. Apodaca Gorostidi, I. Autoestima y locus de control en una muestra de Proyecto Hombre. Adicciones. 1993. 5, 4: 349-357.

26. Lila, M.S.; Musitu, G.; Molpereces, M.A. Familia y autoconcepto. En G. Musitu y P. Allat (Eds.). Psicosociología de la familia.. Albatros. Valencia. 1994.

27. Higgins, E.T. The «self-digest»: self-knowledge serving self-regulatory functions. Journal of Personality and Social Psychology. 1996. 71: 1062-1083.

28. Stein, K.F.; Markus, H.R. The role of the self in behavioral change. Journal of Psychotherapy Integration. 1996. 6, 4: 349-384.

29. Mruk, CH. Self-esteem: research, theory and practice. Springer Publishing Company. New York. 1995.

30. Rosenberg, M. Society and adolescent self-image. Princeton University Press. Princeton. 1965.

31. Vallés Lorente, A. Padres, hijos y drogas. Una estrategia de intervención psicológica para la prevención de las drogodependencias. Quaderns de drogodependències núm 1. Consellería de Benestar Social. Generalitat Valenciana. Valencia. 1997.

32. Blascovich, J.; Tomaka, J. Measures of self-steem. En J.P. Robinson, P.R. Shaver; L.S. Wrightsman (Eds.). Measures of personality and social psychological attitudes. Academic Press. 1991.

33. Rotter, J.B. Generalized expectancies for internal versus external control of reinforcement. Psychological Monographs: General and Applied. 1966. 80, (1, Whole $\mathrm{n}^{\circ}$ 609).

34. Beauvois, J.L. La psychologie quotidienne. PUF. París.1985.

35. Phares, E.J. Locus of control in personality. Houghton Mifflin. New York. 1976.

36. Tsiboukli, A. Understanding alcohol use amongst adolescent students at the innes City London Area. Itaca. 1988. III, 3: 65-84.

37. Bandura, A. Self-efficacy: towards a unifying theory of behavioral change. Psychological Review. 1977. 84: 191-215.

38. Beels, C. CH. Social support and schizophrenia. Schizophrenia Bulletin. 1981. 7: 58-72.

39. House, J.J. Work stress and social support. Reading M.A. Addison - Wesley 1981.

40. Dohrenwend, B.P., Shrout, P.E., Link, B.G.; Skodol, A.E. Social psychological risk factors for episodes of schizophrenia. En H. Häfner, W.F. Gattaz y W. Jazarik. Search for the causes of schizophrenia. Springer - Verlag. 1987. 
41. Gore, S. Stress-Buffering functions of social support. An Appraisal and clarification of research models. En stressful life events. Dillons. New York. 1984.

42. Wellman, B. Applying network analysis to the study of support. En B.H. Gottlieb (Ed.) Social network and social support (pp. 171-200) C.A. Sage. Beverly Hills. 1981.

43. Bott, E. Family and social network. Tavistok Publications. London 1971.

44. Pattison, E.M.; Pattison, M.L. Analysis of Schizophrenic Psychosocial Network. Schizophrenia Bulletin. 1981. 7: 135-143.

45. Alonso Castaño, 1. Seguridad e Higiene Laboral en hostelería y restauración. A.M. V Ediciones. Madrid. 1995.

46. Schmidtke, H. Die Ermudung: Symptome, Theorien, Messrersuche. En Bern/Stuttgart Wien. Verlag Hans Huber.1965.

47. Bartley, S.H. What do we call fatigue?. En E. Simonson y P.C. Weiser (Eds.) Psychological aspects and physiological correlates of work and fatigue. Springfield. C.C. Thomas. 1976.

48. Levine, P.H. Chronic Fatigue Syndrome come to age. American Journal of Medicine. 1998. 105, 3A: S2-S6.

49. Wesseley, S.; Chalder, T.; Hirsh, S.; Wallace, P.; Wright, D. The prevalence and morbility of CF and CFS: A prospective primary care study. American Journal of Public Health. 1997. 87: 1499-1555.

50. Arquer, M.L. Carga mental de trabajo: Fatiga. NTP 445 Instituto Nacional de Seguridad e Higiene en el Trabajo. 1997.

51. Newsholme, E.A.; Blomstrand, E.; Ekblom, B. Physical and mental fatigue: Metabolic mechanisms and importance of plasma amino acids. British Medical Bulletin. 1992. 48, 3: 477-495.

52. Court Lobo, J. Síndrome de fatiga crónica: Historia de una controversia. www.escuela.med.puc.cl/publicaciones. 2002.

53. Sharpe, M.; Archard, L.; Banatvala, J. A report chronic fatigue syndrome: Guidelines for research. J. R. Soc. Med. 1991. 84: 118-121.

54. Holmes, G.; Kaplan, J.E.; Gantz, N.M. Chronic fatigue syndrome: A working case definition. Ann. Intern. Med. 1988. 108: 387-389.

55. Calabresse, L. Chronic Fatigue Syndrome. American Family Physician. 1992. 45, 3: 1205-1213.

56. Straus, S.E. History of Chronic Fatigue Syndrome. Rev. Infect. Dis. 1991. 13 (Supp.1): 1-7.

* Psicólogo

Departamento del Servicio de Prevención. Area de Personal. Ayuntamiento de Madrid. 